FERRAZ, H.T. et al. Desenvolvimento folicular e pico de LH em fêmeas Nelore (Bos taurus indicus) com ovulação sincronizada. PUBVET, Londrina, V. 8, N. 22, Ed. 271, Art. 1809, Novembro, 2014.

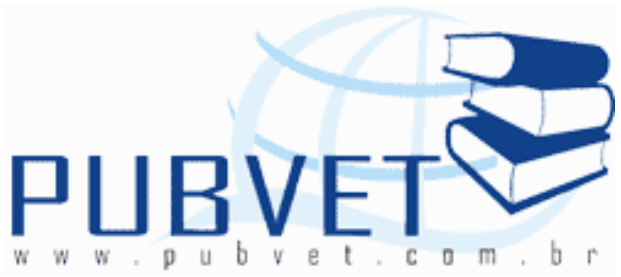

PUBVET, Publicações em Medicina Veterinária e Zootecnia.

\title{
Desenvolvimento folicular e pico de LH em fêmeas Nelore (Bos taurus indicus) com ovulação sincronizada*
}

\footnotetext{
Henrique Trevizoli Ferraz ${ }^{1}$, Benedito Dias de Oliveira Filho² ${ }^{2}$ Maria Lúcia Gambarini ${ }^{2}$, Marco Antônio de Oliveira Viu ${ }^{1}$, Dyomar Toledo Lopes ${ }^{1}$, Juliano Pereira Terra ${ }^{3}$, Lorenna C. Rezende ${ }^{4}$, Ana Paula F. Sousa ${ }^{4}$
}

* Parte da Dissertação de Mestrado do Primeiro Autor

${ }^{1}$ Professor do Curso de Medicina Veterinária. Universidade Federal de Goiás. Campus Jataí (UFG/CAJ). e-mail: htferraz@gmail.com

${ }^{2}$ Professor(a) da Escola de Veterinária e Zootecnia. Universidade Federal de Goiás. Goiânia-GO (EVZ/UFG).

${ }^{3}$ Médico Veterinário. Aluno de Residência em Medicina Veterinária - UFG/CAJ

${ }^{4}$ Médica Veterinária. Autônoma.

\section{Resumo}

Este trabalho foi desenvolvido com o objetivo de se acompanhar a atividade ovariana e o momento do pico pré-ovulatório de LH em novilhas e vacas Nelore submetidas a um protocolo hormonal de sincronização da ovulação com norgestomet (NOR), benzoato de estradiol (BE) e cloprostenol sódico. No experimento 1 , cinco vacas e cinco novilhas receberam aplicação de 2,0 mg de BE e colocação do implante de NOR no dia zero (D0), com retirada no D8 seguida da aplicação de 0,5 mg de cloprostenol. No D9 aplicou-se $1,0 \mathrm{mg}$ de $\mathrm{BE}$, realizando-se acompanhamento ultrassonográfico da atividade ovariana 
FERRAZ, H.T. et al. Desenvolvimento folicular e pico de LH em fêmeas Nelore (Bos taurus indicus) com ovulação sincronizada. PUBVET, Londrina, V. 8, N. 22, Ed. 271, Art. 1809, Novembro, 2014.

desde o início do protocolo até a ovulação. No experimento 2, seis fêmeas de cada categoria foram submetidas ao mesmo tratamento. Vinte e sete horas após a segunda dose de BE amostras de sangue foram coletadas a cada 15 minutos, durante um período de seis horas, para determinação dos níveis séricos de LH. O diâmetro médio do folículo ovulatório foi de $8,02 \pm 0,39 \mathrm{~mm}$ nas novilhas e de $9,10 \pm 0,24 \mathrm{~mm}$ nas vacas $(\mathrm{P}<0,05)$. O pico pré-ovulatório de

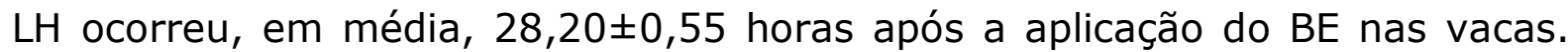
Pôde-se concluir que o desenvolvimento folicular de novilhas e vacas Nelore submetidas à sincronização da ovulação ocorre de maneira semelhante, sendo o folículo ovulatório maior nas vacas. Sugere-se ainda que nas novilhas o pico de $\mathrm{LH}$, provavelmente, ocorra mais precocemente que nas vacas.

Palavras-chave: Benzoato de estradiol, Bovinos, IATF, Progestágenos, Reprodução

\title{
Follicular development and LH peak on Nellore (Bos taurus indicus) females with synchronized ovulation
}

\begin{abstract}
The objective with this work was to monitor ovarian activity and the moment of LH pre-ovulatory peak in Nellore heifers and cows submitted to a hormonal protocol for ovulation synchronization with norgestomet (NOR), estradiol benzoate (EB) and cloprostenol. In experiment 1, five cows and five heifers received application of $2.0 \mathrm{mg}$ of EB and implant of NOR on day zero (D0), with removal on D8 following application of $0.5 \mathrm{mg}$ of cloprostenol. On D9 1.0 $\mathrm{mg}$ of EB was applied, performing ultrasound monitoring of ovarian activity since the beginning of the protocol until ovulation. In experiment 2, six females in each category were submitted to the same treatment. Twenty seven hours after BE blood was collected every 15 minutes, over a six hours period, for determination of $\mathrm{LH}$ serum levels. The mean diameter of the ovulatory follicle was $8.02 \pm 0.39 \mathrm{~mm}$ in heifers and $9.10 \pm 0.24 \mathrm{~mm}$ in cows $(P<0.05)$. The LH pre-ovulatory peak occurred, on average, $28.20 \pm 0.55$ hours after the
\end{abstract}


FERRAZ, H.T. et al. Desenvolvimento folicular e pico de LH em fêmeas Nelore (Bos taurus indicus) com ovulação sincronizada. PUBVET, Londrina, V. 8, N. 22, Ed. 271, Art. 1809, Novembro, 2014.

application of EB in cows. We can conclude that follicular development in Nellore heifers and cows submitted to ovulation synchronization occurs in a similar way, being the largest ovulatory follicle in cows. It's suggested that in heifers LH peak probably occurs earlier than in cows.

Keywords: Bovine, Estradiol benzoate, Progestins, Reproduction, TAI

\section{INTRODUÇÃO}

O diâmetro máximo do folículo pré-ovulatório em zebuínos está em torno de 10,00 a 12,00 mm (BORGES et al., 2003), menor do que o frequentemente relatado para taurinos (WOLFENSON et al., 2004). MAIO et al. (2006), trabalhando com novilhas Nelore sincronizadas, encontraram intervalo médio de 22,40 horas entre a aplicação do benzoato de estradiol (BE) e o pico préovulatório de LH.

O Centro-Oeste brasileiro possui um rebanho bovino de aproximadamente 72 milhões de cabeças, com mais de $80 \%$ de sangue zebuíno, onde predomina a raça Nelore. Dentre as biotecnologias aplicáveis à pecuária comercial destacam-se a inseminação artificial (IA) e a inseminação artificial em tempo fixo (IATF), sendo estas estratégias economicamente viáveis que propiciam a melhoria do rebanho por meio do uso do sêmen de touros comprovadamente superiores. No entanto ainda são escassos os estudos sobre a fisiologia reprodutiva de fêmeas Nelore submetidas a protocolos hormonais para sincronização da ovulação no Brasil.

Neste sentido, desenvolveu-se este trabalho com o objetivo de acompanhar a atividade ovariana e o momento do pico pré-ovulatório de $\mathrm{LH}$ em novilhas e vacas Nelore submetidas a um protocolo hormonal de sincronização da ovulação para realização da IATF.

\section{MATERIAL E MÉTODOS}

Este estudo foi desenvolvido em abril de 2006 na Fazenda Santa Rosa, localizada no município de Caçu, região sudoeste do Estado de Goiás, Brasil. No experimento 1 , para o acompanhamento da atividade ovariana e do 
FERRAZ, H.T. et al. Desenvolvimento folicular e pico de LH em fêmeas Nelore (Bos taurus indicus) com ovulação sincronizada. PUBVET, Londrina, V. 8, N. 22, Ed. 271, Art. 1809, Novembro, 2014.

momento da ovulação, foram utilizadas cinco novilhas com peso mínimo de $300 \mathrm{~kg}$ e idade entre 24 e 30 meses, e cinco vacas entre a segunda e a quinta ordem de parição com, pelo menos, 60 dias pós-parto e bom escore de condição corporal. Durante o período experimental as fêmeas foram mantidas em pastagem de Brachiaria brizantha cv. Marandú, próximas ao curral de manejo. Todos os animais foram submetidos ao seguinte protocolo: aplicação, via intramuscular (IM), de 2,0 mg de BE no momento da implantação auricular do norgestomet (NOR), sendo este considerado o dia zero (D0) do protocolo. Após oito dias (D8), juntamente com a retirada do implante, realizou-se a aplicação, IM, de 0,50 mg de cloprostenol sódico. Após 24 horas (D9) aplicouse, IM, 1,0 mg de BE.

A atividade ovariana foi acompanhada por meio de exames ultrassonográficos, em intervalos de 24 horas, desde a colocação do implante auricular de NOR (D0) até sua retirada (D8). A partir de então e até o momento da aplicação da segunda dose de BE (D9), realizou-se a ultrassonografia a cada 12 horas. Após a segunda dose de BE estas avaliações foram feitas a cada seis horas, até que se verificasse a ovulação do folículo dominante (FD). O momento da ovulação foi estimado pelo intervalo médio entre a observação do maior diâmetro do FD e seu desaparecimento na imagem ultrassonográfica do exame seguinte. Seis dias após a ovulação realizou-se novo exame para verificação da presença do corpo lúteo (CL). Para mensuração do tempo necessário para o início de uma nova onda de crescimento folicular, considerou-se o surgimento do primeiro folículo com diâmetro maior ou igual a três milímetros após o início do protocolo hormonal (BARROS et al., 2000). Os exames foram feitos com auxílio do aparelho Pie Medical 480, equipado com transdutor retal bifrequencial de 5,0/7,5 MHz. No momento do exame o transdutor era movimentado sobre a superfície dos ovários e, quando necessário, congelava-se a imagem do monitor para a mensuração do diâmetro dos folículos ou do CL. Durante cada exame eram confeccionados diagramas com o posicionamento relativo das estruturas avaliadas. 
FERRAZ, H.T. et al. Desenvolvimento folicular e pico de LH em fêmeas Nelore (Bos taurus indicus) com ovulação sincronizada. PUBVET, Londrina, V. 8, N. 22, Ed. 271, Art. 1809, Novembro, 2014.

Os dados gerados foram analisados usando-se os procedimentos do pacote computacional WINSTAT for Excel (FITCH, 2006), primeiramente realizando-se análises de crítica e consistência (frequências, distribuições de frequências e homogeneidade de variâncias). Procedeu-se então a comparação entre as médias das variáveis: diâmetro dos folículos ovulatórios; intervalo entre o início do protocolo e surgimento de uma nova onda de crescimento folicular; entre a retirada do implante de NOR e a ovulação; e entre a aplicação de $B E$ e a ovulação, através do procedimento independent $t$-test do referido programa.

No experimento 2, para a avaliação da frequência de pulsos e da amplitude do pico pré-ovulatório de LH, foram utilizadas seis novilhas e seis vacas, provenientes de um grupo de 50 animais de onde também foram sorteados aqueles pertencentes ao experimento 1 e que, portanto, foram submetidas aos mesmos critérios de seleção (idade, peso, escore de condição corporal, dias pós-parto e ordem de partos). Esse experimento teve início logo após o término dos exames ultrassonográficos do experimento anterior, sendo utilizado nos animais mesmo protocolo de sincronização da ovulação. Foram realizadas colheitas de sangue iniciadas 27 horas após a aplicação da segunda dose de $B E$, sendo repetidas a cada 15 minutos por um período de seis horas, ou seja, entre a $27^{\mathrm{a}}$ e a $33^{\mathrm{a}}$ hora após a aplicação do $B E$, totalizando assim 24 observações. Os animais foram mantidos nos troncos de contenção e o sangue colhido por punção da veia jugular, com auxílio de agulhas descartáveis 40×12 $\mathrm{mm}$. O material obtido foi armazenado em tubos de ensaio identificados e mantidos na posição inclinada no interior de uma caixa térmica com gelo. Posteriormente procedeu-se a centrifugação a $1520 \mathrm{~g}$ por 15 minutos, sendo o soro obtido armazenado a $-20^{\circ} \mathrm{C}$. As amostras congeladas do soro sanguíneo foram remetidas ao Laboratório de Endocrinologia da Escola de Veterinária da UNESP - Campus de Araçatuba-SP, para a determinação dos níveis séricos de LH pelo método de radioimunoensaio com kits desenvolvidos na própria instituição. 
FERRAZ, H.T. et al. Desenvolvimento folicular e pico de LH em fêmeas Nelore (Bos taurus indicus) com ovulação sincronizada. PUBVET, Londrina, V. 8, N. 22, Ed. 271, Art. 1809, Novembro, 2014.

Os resultados obtidos no experimento 2 foram tabulados em planilhas eletrônicas, procedendo-se então análise descritiva utilizando-se o procedimento FREQ do pacote computacional SAS (2000).

\section{RESULTADOS E DISCUSSÃO}

No experimento 1, conforme demonstrado na Tabela 1, o intervalo médio entre o início do protocolo e o surgimento de uma nova onda de crescimento

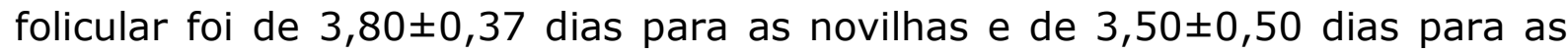
vacas. Outros autores relataram intervalos entre três e seis dias para vacas e novilhas submetidas a este tratamento (GARCIA \& SALAHEDDINE, 2001; CAVALIERI et al., 2002; MARTINEZ et al., 2005; MELO, 2009). Adicionalmente, BARROS et al. (2000) encontraram intervalo médio de 4,36 dias entre o início do protocolo com BE + Progesterona e o surgimento de uma nova onda folicular em vacas Nelore. No presente estudo a ovulação ocorreu, em média,

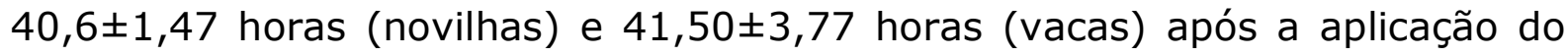
BE no D9 do protocolo. BARROS et al. (2000) relataram intervalo de 44,16 horas entre a aplicação do BE e a ovulação em vacas Nelore. CAVALIERI et al. (2002), trabalhando com novilhas meio-sangue zebuíno de corte, observaram intervalo de $50,4 \pm 1,7$ horas entre a aplicação do BE e a ovulação. Segundo MARTINEZ et al. (2005), este mesmo intervalo foi de 53,3 $\pm 1,9$ horas em vacas taurinas. Os valores obtidos neste estudo estão abaixo dos relatados pela literatura, provavelmente devido ao menor diâmetro do FD observado neste experimento. Para os intervalos anteriormente discutidos não se verificou diferença $(P>0,05)$ entre novilhas e vacas pelo independent $t$-test.

O diâmetro médio do folículo ovulatório das novilhas foi de $8,02 \pm 0,39$

$\mathrm{mm}$ (Tabela 1). Este valor é menor que os $11,60 \mathrm{~mm}$ verificados por CAVALIERI et al. (2002) em novilhas Bos taurus indicus sincronizadas com dispositivo intravaginal de progesterona e aplicação de BE 24 horas após a remoção deste. Nestas mesmas condições, GARCIA e SALAHEDDINE (2001) verificaram média de $13,60 \mathrm{~mm}$ para o folículo ovulatório de novilhas da raça Holandesa. Os menores valores encontrados neste experimento devem-se, 
FERRAZ, H.T. et al. Desenvolvimento folicular e pico de LH em fêmeas Nelore (Bos taurus indicus) com ovulação sincronizada. PUBVET, Londrina, V. 8, N. 22, Ed. 271, Art. 1809, Novembro, 2014.

provavelmente, à dose excessiva de BE para esta categoria, uma vez que, segundo VOGG et al. (2004), 0,50 mg de BE deve ser a dose máxima utilizada em novilhas para estimular a ovulação, sem afetar o tamanho e a qualidade do folículo ovulatório.

TABELA 1 - Intervalo médio \pm desvio padrão ( $\mu \pm D P)$ e coeficiente de variação (CV) entre o início do protocolo e o surgimento de uma nova onda folicular (IPNO) e entre a aplicação do BE e a ovulação (BE-OV); e diâmetro máximo (DMAX), mínimo (DMIN), médio \pm desvio padrão $(\mu \pm D P)$ e coeficiente de variação (CV) do folículo ovulatório de novilhas e vacas Nelore submetidas ao protocolo BE-NOR-PGF2a-BE

\begin{tabular}{|c|c|c|c|c|}
\hline \multirow{2}{*}{ Intervalos* } & \multicolumn{2}{|c|}{ Novilhas } & \multicolumn{2}{|c|}{ Vacas } \\
\hline & $\mu \pm D P$ & CV(\%) & $\mu \pm D P$ & CV(\%) \\
\hline IP-NO (dias) & $3,80 \pm 0,37$ & 22,01 & $3,50 \pm 0,50$ & 28,57 \\
\hline BE-OV (horas) & $40,6 \pm 1,47$ & 8,09 & $41,50 \pm 3,77$ & 18,19 \\
\hline \multirow{2}{*}{ Variável } & \multicolumn{4}{|c|}{ Folículo Ovulatório** } \\
\hline & \multicolumn{2}{|c|}{ Novilhas } & \multicolumn{2}{|c|}{ Vacas } \\
\hline $\operatorname{DMAX}(\mathrm{mm})$ & \multicolumn{2}{|c|}{8,40} & \multicolumn{2}{|c|}{9,30} \\
\hline DMIN (mm) & \multicolumn{2}{|c|}{7,40} & \multicolumn{2}{|c|}{8,70} \\
\hline$\mu \pm \mathrm{DP}(\mathrm{mm})$ & \multicolumn{2}{|c|}{$8,02 \pm 0,39^{a}$} & \multicolumn{2}{|c|}{$9,10 \pm 0,24^{b}$} \\
\hline CV $(\%)$ & \multicolumn{2}{|c|}{4,94} & \multicolumn{2}{|c|}{2,69} \\
\hline
\end{tabular}

*Não houve diferença $(\mathrm{P}>0,05)$ pelo indepedent $t$-test

$* *$ Letras diferentes na mesma linha indicam diferença pelo independent $t$-test $(P<0,05)$

O estudo da dinâmica folicular nas vacas mostrou que o diâmetro médio

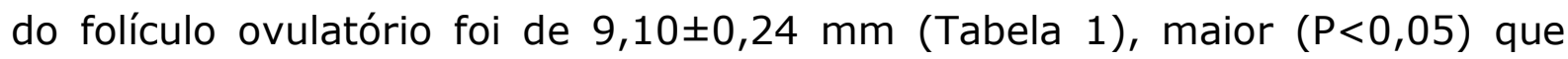
aquele observado nas novilhas. FIGUEIREDO et al. (1997) encontraram valores entre 11,00 e 12,00 mm para os folículos pré-ovulatórios de vacas Nelore durante o ciclo estral fisiológico. Adicionalmente, MELO (2009) observou 
FERRAZ, H.T. et al. Desenvolvimento folicular e pico de LH em fêmeas Nelore (Bos taurus indicus) com ovulação sincronizada. PUBVET, Londrina, V. 8, N. 22, Ed. 271, Art. 1809, Novembro, 2014.

diâmetro médio do folículo pré-ovulatório de 15,90 mm em vacas Braford submetidas ao mesmo protocolo hormonal do presente experimento. Os baixos valores encontrados para o diâmetro do folículo ovulatório neste estudo podem ter ocorrido em função do manejo estressante a que os animais foram submetidos, devido ao exame ultrassonográfico diário em fêmeas que, até então, vinham sendo criadas de maneira extensiva, interferindo na liberação das gonadotrofinas e no desenvolvimento dos folículos, assim como relatado por SMITH e DOBSON (2002).

No experimento 2, em apenas uma das seis novilhas e em quatro das seis vacas, pôde-se observar o pico pré-ovulatório de LH nas amostras de sangue colhidas entre a $27^{\mathrm{a}}$ e a $33^{\mathrm{a}}$ hora após a segunda dose de BE (Figuras 1 e 2).

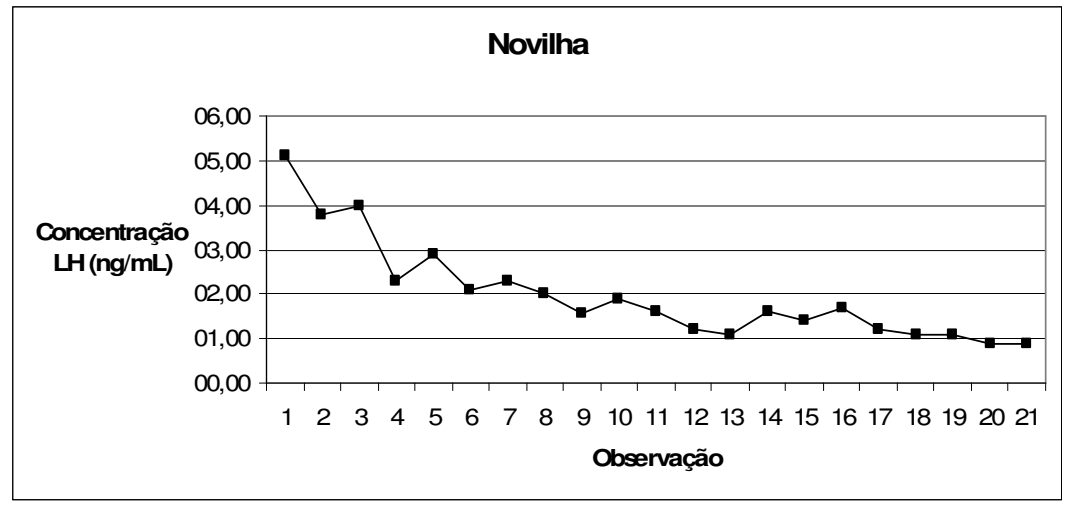

FIGURA 1 - Pico pré-ovulatório de LH na novilha Nelore submetidas ao protocolo BE-NOR-PGF2a-BE, em observações (coletas de sangue) realizadas a cada 15 minutos, 27 horas após aplicação de 1,0 mg de BE 
FERRAZ, H.T. et al. Desenvolvimento folicular e pico de LH em fêmeas Nelore (Bos taurus indicus) com ovulação sincronizada. PUBVET, Londrina, V. 8, N. 22, Ed. 271, Art. 1809, Novembro, 2014.

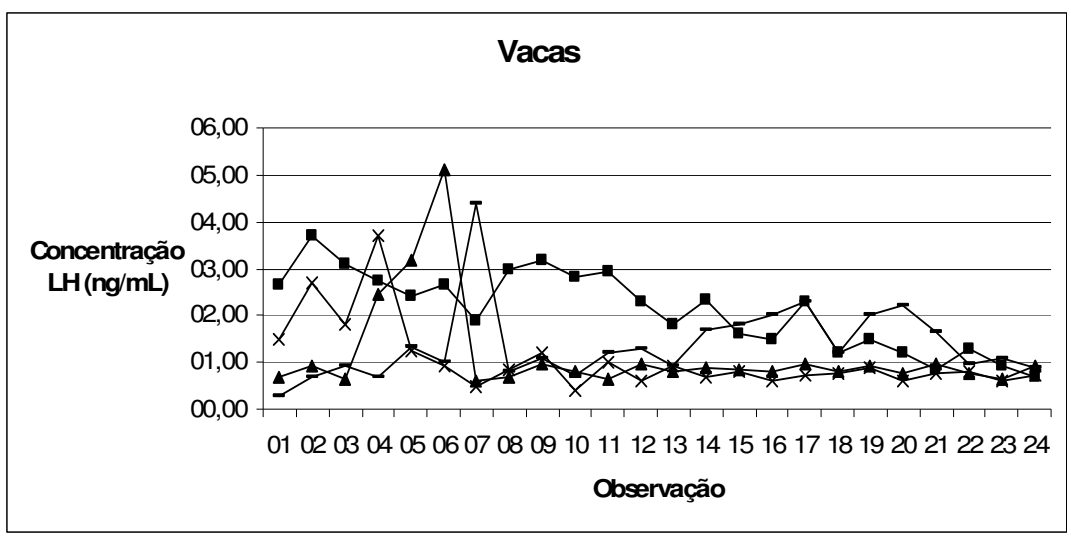

FIGURA 2 - Pico pré-ovulatório de LH nas vacas Nelore submetidas ao protocolo BE-NOR-PGF2a-BE, em observações (coletas de sangue) realizadas a cada 15 minutos, 27 horas após aplicação de 1,0 mg de BE

Na novilha o pico foi verificado logo na primeira colheita, ou seja, 27,00 horas após a indução da ovulação. Já para as vacas este ocorreu, em média, $28,20 \pm 0,55$ horas após a aplicação do BE. MAIO et al. (2006), trabalhando com novilhas Nelore sincronizadas com o mesmo protocolo utilizado neste experimento, encontraram intervalo médio de 22,40 horas entre a aplicação do BE e o pico pré-ovulatório de LH, com variação de 18 a 25 horas. Utilizando protocolo hormonal semelhante, LAMMOGLIA et al. (1998) verificaram intervalo de 20 horas entre aplicação de $0,75 \mathrm{mg}$ de $\mathrm{BE}$ e o pico de LH em novilhas Bos taurus taurus e de 24 horas entre a aplicação de 1,0 mg de BE e o pico deste hormônio em vacas do mesmo grupamento genético.

A grande variação no momento do pico de LH após o uso do BE e o fato deste ocorrer, provavelmente, mais precocemente em novilhas, pode explicar a não verificação do pico pré-ovulatório de LH em todos os animais estudados e a menor frequência de observação deste nessa categoria no intervalo em que foram colhidas as amostras de sangue. Dentre os animais em que se observou o pico pré-ovulatório de $\mathrm{LH}$, a amplitude máxima verificada foi, em média, de 4,58 $\pm 0,67 \mathrm{ng} / \mathrm{mL}$. MAIO et al. (2006) relataram a concentração sérica média de $14,32 \mathrm{ng} / \mathrm{mL}$ de LH para o máximo valor observado em novilhas Nelore, com variação de 6,94 a 20,0 ng/mL. AMBROSE et al. (2005) verificaram 
FERRAZ, H.T. et al. Desenvolvimento folicular e pico de LH em fêmeas Nelore (Bos taurus indicus) com ovulação sincronizada. PUBVET, Londrina, V. 8, N. 22, Ed. 271, Art. 1809, Novembro, 2014.

concentração média de $9,30 \mathrm{ng} / \mathrm{mL}$ no pico de LH de novilhas Holandesas, com valores entre 4,60 e $11,80 \mathrm{ng} / \mathrm{mL}$. A concentração média de LH encontrada neste experimento provavelmente se deva ao estresse do manejo a que animais de criação extensiva foram submetidos no momento das colheitas de sangue, interferindo na amplitude do pico de LH, assim como discutido por SMITH e DOBSON (2002), devido à ação deletéria dos corticosteróides endógenos, secretados em condições estressantes, interferindo no eixo hipotálamo-hipófise-adrenal.

Nos animais em que não se observou o pico de LH foi possível determinar as concentrações médias deste hormônio durante sua liberação pulsátil. Para as novilhas estas foram de $0,90 \pm 0,03 \mathrm{ng} / \mathrm{mL}$ e para as vacas de $0,86 \pm 0,03 \mathrm{ng} / \mathrm{mL}$. CUPP et al. (1995) relataram valores médios de LH de 0,77 $\mathrm{ng} / \mathrm{mL}$ durante o diestro de vacas Bos taurus taurus de corte. MARTINEZ et al. (2005) verificaram, em vacas Hereford tratadas com $17 \beta$ estradiol e dispositivos intravaginais de progesterona, concentrações médias de 0,80 $\mathrm{ng} / \mathrm{mL}$ de LH. Portanto, as concentrações médias da liberação pulsátil de LH nas novilhas e vacas do presente experimento encontram-se próximas às descritas na literatura para essas categorias.

\section{CONCLUSÕES}

Os resultados obtidos neste estudo permitem concluir que o folículo ovulatório encontrado nas vacas é maior que o observado nas novilhas. Sugere-se ainda que nas novilhas o pico pré-ovulatório de LH, provavelmente, ocorra mais precocemente que nas vacas.

\section{REFERÊNCIAS BIBLIOGRÁFICAS}

AMBROSE J.D.; J.P. KASTELIC; R. RAJAMAHERNDRAN; M. AALI; N. DINN. Progesterone (CIDR) based timed AI protocols using GnRH, porcine LH or estradiol cypionate for dairy heifers: ovarian and endocrine responses and pregnancy rates. Theriogenology, v. 64, p. 1457-1474, 2005. 
BARROS, C.M.; M.P.B. MOREIRA.; R.A., FIGUEIREDO.; A.B. TEIXEIRA.; L.A. TRINCA. Synchronization of ovulation in beef cows (Bos indicus) using GnRH, PGF2a and estradiol benzoate. Theriogenology, v. 53, p. 1121-1134, 2000.

BORGES, A.M.; TORRES, C.A.A.; RUAS, J.R.M.; ROCHA JUNIOR, V.R.; CARVALHO, G.R.; FONSECA, J.F.; MARCATTI NETO, A.; ASSIS, A.J. Características da dinâmica folicular e regressão luteal de vacas das raças Gir e Nelore após tratamento com cloprostenol sódico. Revista Brasileira de Zootecnia, v.32, n.1, p.85-92, 2003.

CAVALIERI, J.; C. COLEMAN.; H. RODRIGUES.; K.L. MACMILLAN.; L.A. FITZPATRICK. The effect of timing of administration of oestradiol benzoate on characteristics of oestrus, timing of ovulation and fertility in Bos indicus heifers synchronized with a progesterone releasing intravaginal insert. Australian Veterinary Journal, v. 80, p. 217-223, 2002.

CUPP A.S.; T.T. STUMPF.; F.N. KOJIMA.; L.A. WERTH.; M.W. WOLFE.; M.S. ROBERSON.; R.J. KITTOK.; J.E. KINDER. Secretion of gonadotrophins change during the luteal phase of the bovine oestrus cycle in the absence of corresponding changes in progesterone or $17 \beta$ estradiol. Animal Reproduction Science, v. 37, p. 109-119, 1995.

FIGUEIREDO, R.A.; C.M. BARROS.; O.L. PINHEIRO.; J.M.P. SOLER. Ovarian follicular dynamics in Nellore breed (Bos indicus) cattle. Theriogenology, v. 47, p. 1489-1505, 1997.

FITCH, R.K. User's manual: WinSTAT for Excel. R. Fitch Software. Bad Krozingen. Germany, 2006.

GARCIA, A.; M. SALAHEDDINE. Effect of oestrus synchronization with estradiol 17a and progesterone on follicular wave dynamics in dairy heifers. Reproduction in Domestic Animals, v. 36, p. 301-307, 2001.

LAMMOGLIA, M.A.; R.E. SHORT.; S.E. BELLOWS.; R.A. BELLOWS.; M.D. MACNEIL.; HAFS, H.D. Induced and synchronization estrus in cattle: dose titration of estradiol benzoate in peripuberal heifers and postpartum cows after treatment with an intravaginal progesterone releasing insert and prostaglandinF2a. Journal Animal Science, v. 76, p. 1662-1670, 1998.

MAIO, J.R.G.; G.A.F. SANDOVAL.; E.D.F. SOUZA.; G.P.NOGUEIRA.; R.S. CIPRIANO.; F. PERECIN.; J.M. GARCIA. Concentração sérica de LH em vacas nelore ciclando ou ovariectomizadas submetidas ao tratamento com 2,0 $\mathrm{mg}$ de benzoato de estradiol. Acta Scientiae Veteninariae, v. 34 (supl. 1), p. 360, 2006.

MARTINEZ, M.F.; J.P. KASTELIC.; G.A. BÓ.; M. CACCIA.; R.J. MAPLETOFT. Effects of oestradiol and some of its esters on gonadotrophin release and ovarian follicular dynamics in CIDRtreated beef cattle. Animal Reproduction Science, v.86, p. 37-52, 2005.

MELO, L. C. Dinâmica folicular de vacas de corte tratadas com três protocolos de sincronização da ovulação. 2009. 41 f. Dissertação (Mestrado em Ciências Veterinárias) Faculdade de Veterinária, Universidade Federal do Rio Grande do Sul, Porto Alegre.

SAS. 2000. Statistical Analysis System. User's guide: Statistic. v. 8. SAS Institute. Cary. 295 p., 2000.

SMITH, R.F.; H. DOBSON. Hormonal interactions within the hypothalamus and pituitary with respect to stress and reproduction in sheep. Domestic of Animal Endocrinology, v. 23, p. 75-85, 2002. 
VOGG, G.; C.J.H. SOUZA., C.M. JAUME.; J.C.F. MORAES. Utilidade do benzoato de estradiol após suplementação com gestágeno na sincronização de cios de novilhas de corte. Acta Scientiae Veterinariae, v. 32, p. 41-46, 2004.

WOLFENSON, D.; INBAR, G.; ROTH, Z.; KAIM, M; BLOCK, A.; BRAW-TAL, R. Follicular dynamics and concentrations of steroids and gonadotropins in lactating cows and nulliparous heifers. Theriogenology, v.62, p.1042-1055, 2004. 